\title{
Reflective-SOA Fiber Cavity Laser as Directly Modulated WDM-PON Colorless Transmitter
}

\author{
Simon A. Gebrewold, Lucia Marazzi, Paola Parolari, Romain Brenot, Seán P. Ó. Dúill, Romain Bonjour, \\ David Hillerkuss, Christian Hafner, and Juerg Leuthold, Fellow, IEEE
}

\begin{abstract}
Reflective semiconductor optical amplifier (RSOA) fiber cavity lasers are attractive colorless, self-seeded, self-tuning, and directly modulatable sources for passive optical networks (PONs). They comprise of an RSOA in the optical network unit as the active element, a distribution fiber as the laser cavity, a waveguide grating router, and a common reflective mirror with the latter two positioned at the remote node. In this paper, we introduce a model and perform simulations to elucidate the recently discovered successful operation of this new PON source. The results are in agreement with experiments; the formation of a narrow laser spectrum with a relatively constant output power is seen despite a relatively broad passband window of the waveguide grating router. We further study mode competition and mode partition noise. It is shown that proper chromatic dispersion management can overcome mode partition noise limitations. The quality of the RSOA fiber cavity laser does not degrade when being directly modulated and as a result these highly multimode lasers offer an economic way to transport Gbit/s upstream data over kilometers of fiber in a wavelength division multiplexing-PON.
\end{abstract}

Index Terms-Colorless transmitter, reflective semiconductor optical amplifier (RSOA) fiber cavity laser (FCL), self-seeding, semiconductor optical amplifier, wavelength division multiplexingpassive optical network (WDM-PON).

\section{INTRODUCTION}

$\mathbf{R}$ EFLECTIVE semiconductor optical amplifiers (RSOAs) are already deployed as colorless modulators in wavelength division multiplexing (WDM) passive optical networks (PONs). They further hold promise to not only act as colorless modulators but also to act as colorless laser sources if the access network fiber trunk is used as a laser cavity [1], [2].

RSOAs so far are largely deployed as externally seeded upstream (US) transmitters. In these transmitters, the RSOA

Manuscript received December 7, 2013; revised February 9, 2014; accepted February 10, 2014. Date of publication February 20, 2014; date of current version April 22, 2014. This work was supported by the European FP7 project ERMES under Grant 2885422012.

S. A. Gebrewold, R. Bonjour, D. Hillerkuss, C. Hafner, and J. Leuthold are with the Institute of Electromagnetic Field, ETH Zurich, 8092 Zurich, Switzerland (e-mail: sgebrewold@ethz.ch; rbonjour@ethz.ch; dhillerkuss@ethz.ch; christian.hafner@ifh.ee.ethz.ch; leuthold@ethz.ch).

L. Marazzi and P. Parolari are with the PoliCom, Politecnico di Milano, Milan 20133, Italy (e-mail: lucia.marazzi@ polimi.it; paola.parolari@polimi.it).

R. Brenot is with the III-V Lab, a joint lab of Alcatel-Lucent Bell Labs France, Thales Research and Technology and CEA Leti, 91460 Marcoussis, France (e-mail: romain.brenot@3-5lab.fr).

S. P. Ó. Dúill is with the RINCE Institute, Dublin City University, Dublin 9, Ireland (e-mail: sean.oduill@dcu.ie).

Color versions of one or more of the figures in this paper are available online. modulates a light that originates from a different point in the network. And even though a common external broadband seeding source has shown to work well, the reach and speed of such schemes are limited by amplified spontaneous emission (ASE) beat noise and chromatic dispersion (CD) due to the broad spectrum [3]. In addition, they require high power and broadband light sources, which makes them cost-inefficient. Alternatively, RSOA based self-seeded WDM-PON US transmitters were suggested to eliminate the external seeding source. Initial implementations of self-seeded sources employed feedback mechanisms including fiber Bragg gratings, [4], [5]. Unfortunately, these methods require separate and accurately tuned wavelength selective components in every optical network unit (ONU), thus preventing colorless operation and increasing the complexity and cost.

Recently, a more simple and cost-effective network topology was introduced that incorporates self-seeded RSOA-fiber cavity lasers (RSOA-FCLs) [1], [6], [7]. The resonant laser cavity is formed by the distribution network between the RSOA's reflective facet and a mirror at the remote node $(\mathrm{RN})$. The gain of the cavity is provided by the RSOA whereas the waveguide grating router (WGR) at the RN automatically selects the operating wavelength. The WGR thus not only confines the optical bandwidth of the RSOA-FCL but also allows for an automatic wavelength assignment that is easily scalable so that many users within the network can transmit information at pre-defined wavelength. The number of users is limited by the number of output ports of the WGR.

For proper functioning of the overall system, the RSOA in the cavity should perform three operations. Firstly, the gain has to compensate the overall cavity losses for the system to lase. Secondly, the RSOA should offer a sufficient modulation bandwidth. Thirdly, the residual modulation on the optical field in the US path of the cavity must be nonlinearly suppressed upon return to the RSOA to provide a clean carrier for new US data transmission. Properly designed and operated RSOAs are able to offer the above three functionalities simultaneously. In particular, large gain bulk RSOAs can meet these specifications when the active region length and the confinement factor are appropriately optimized [8]. So far, the modulation cancellation capacity of saturated RSOAs has been demonstrated theoretically and experimentally in [9] and [10]. Also, the electrooptical bandwidth of quantum-well RSOAs have been explored and $10 \mathrm{Gbit} / \mathrm{s}$ direct modulation has been demonstrated [11]. The entire RSOA-FCL system has been experimentally demonstrated in a WDM-PON for beyond $2.5 \mathrm{Gbit} / \mathrm{s}$ US transmission [6], [12]. 
Despite the amount of experimental demonstrations, so far no theoretical and numerical treatment of the RSOA-FCL system has been offered, thus the working principle is poorly understood.

In this paper we present a theoretical investigation, with supporting experimental results, of the RSOA-FCL with a cavity formed by the distribution fibers in the order of a few meters to tens of kilometers length. The RSOA-FCL excites a large numbers of longitudinal modes due to its extra-long cavity. In order to properly simulate the interaction of these modes, we construct a bidirectional RSOA model that accounts for: 1) all the counter-propagating laser modes; 2) the dynamic carrier injection in order to simulate the direct modulation capability and 3) spontaneous and stimulated emission. Using this model, we discuss the effects of $\mathrm{CD}$ and mode partition noise. We show how the RSOA in a fiber cavity can be used as directly modulatable, colorless, self-seeded US transmitter. It will be shown, how the interplay between the nonlinear RSOA together with a properly designed fiber cavity suppresses the intensity fluctuations arising from the multi-mode nature of the laser and also allows for efficient cancelation of the modulation component of the recirculating signal in the FCL. In particular, the performance of the RSOA-FCL with cavity lengths up to $10 \mathrm{~km}$ as a directly modulated US transmitter at $2.5 \mathrm{Gbit} / \mathrm{s}$ is discussed.

\section{WDM-PON ARCHITECTURE}

Before proceeding with the analysis of the RSOA-FCL, we briefly review the RSOA-FCL's PON topology. The topology of a WDM-PON access network with a central office $(\mathrm{CO})$ connected to the RN and the ONUs through the feeder and distribution fibers, respectively, is shown in Fig. 1. The ONU only comprises of an RSOA, a downstream receiver (DS Rx) and a C/L-band WGR for the C-band signal in the US and the L-band signal in the DS, respectively. The RSOA serves as an active medium of the fiber laser cavity, which is formed by the RSOA mirror on one side and the Faraday rotator mirror (FRM) at the $\mathrm{RN}$ on the other side. The cyclic WGR multiplexer at the RN acts as a frequency selective filter through which the laser "self-tunes" to the particular WGR connection port. The RSOA further allows encoding of the user US data by direct modulation of the RSOA injection current.

The Faraday rotator (FR) in the ONU and the RN in the cavity are needed to enable lasing even when the RSOA should have some polarization gain dependence [13].

\section{RSOA-FIBER LASER MODELING}

This section deals with the overall mathematical realization of the RSOA-FCL model. Firstly, a multimode and bidirectional travelling wave RSOA model is presented. Then we introduce models for the passive cavity, which consists of an optical fiber and an optical bandpass filter (OBPF). Finally, the two are combined to form the entire RSOA-FCL system.

\section{A. RSOA Model}

The RSOA model is based on a bidirectional SOA implementation [9], [14]. Fig. 2 depicts the simulation procedure where the RSOA is sliced into $m$ spatial sections. The temporally resolved carrier density and gain are calculated within each section. The optical field propagation is described in terms of travelling wave equations, and is solved for all the counter-propagating multimode contributions to the signal and ASE fields. The consideration of ASE is necessary to generate an RSOA-FCL lasing field that is self-seeded from the ASE and also to accurately account for RSOA gain saturation.

The carrier density rate equation is given by

$$
\frac{\partial N(z, t)}{\partial t}=\frac{I}{q V}-R_{\text {spon }}(z, t)-R_{\text {stim }}(z, t)
$$

where $N(z, t)$ is the carrier density and $I$ is the bias current. $R_{\text {spon }}$ and $R_{\text {stim }}$ represent carrier depletion due to spontaneous and stimulated emission, respectively. The expression for $R_{\mathrm{spon}}$ is given by

$$
\begin{aligned}
R_{\text {spon }}(z, t) & =\frac{N(z, t)}{\tau_{c}} \\
& =\mathrm{AN}(z, t)+\mathrm{BN}(z, t)^{2}+\mathrm{CN}(z, t)^{3}
\end{aligned}
$$

where $\tau_{c}$ is the effective carrier life time which is related to the nonradiative recombination rate $A$, spontaneous recombination coefficient $B$ and Auger recombination coefficient $C . R_{\text {stim }}$ includes the carrier depletion due to the signal $r_{s}(z, t)$, and the ASE $r_{\text {ase }}(z, t)$ and is given by

$$
\begin{aligned}
R_{\text {stim }}(z, t) & =\frac{1}{d w} \frac{\Gamma\left(r_{s}(z, t)+r_{\text {ase }}(z, t)\right)}{h c\left(1+\varepsilon_{\mathrm{NL}} P_{\mathrm{tot}}(z, t)\right)} \\
r_{s}(z, t) & =\left|\sum_{k}\left(\sqrt{\lambda_{k} g_{k}(N)}\left[E_{k}^{+}(z, t)+E_{k}^{-}(z, t)\right]\right)\right|^{2} \\
r_{\text {ase }}(z, t) & =2\left|\sum_{j}\left(\sqrt{\lambda_{j} g_{j}(N)}\left[\varepsilon_{j}^{+}(z, t)+\varepsilon_{j}^{-}(z, t)\right]\right)\right|^{2}
\end{aligned}
$$

where we define $E_{k}^{+}(z, t)$ and $E_{k}^{-}(z, t)$ as the forward and backward propagating complex amplitude of the $k$ th longitudinal mode. Similarly $\varepsilon_{j}^{+}(z, t)$ and $\varepsilon_{j}^{-}(z, t)$ are the fields of the $j$ th ASE mode. The modes can be further decomposed using amplitude and phase coefficients such that

$$
\begin{aligned}
E_{k}^{ \pm}(z, t) & =\tilde{E}_{k}^{ \pm}(z) e^{j 2 \pi v_{k} t} \\
\varepsilon_{k}^{ \pm}(z, t) & =\tilde{\varepsilon}_{j}^{ \pm}(z) e^{j 2 \pi v_{j} t}
\end{aligned}
$$

where $\Gamma, d w, \varepsilon_{\mathrm{NL}}$, and $h$ are the confinement factor, effective area of the RSOA active region, the nonlinear gain coefficient and the Plank's constant, respectively. The indexes $k$ and $j$ refer to the longitudinal modes for the signal and ASE, respectively. For the considered simulation bandwidth of $4 \mathrm{~nm}$, this paper assumes a linear gain as

$$
g(N(z, t))=a_{0}\left(N(z, t)-N_{0}\right)
$$

where $a_{0}$ is the differential gain coefficient and $N_{0}$ is the carrier density at transparency. 


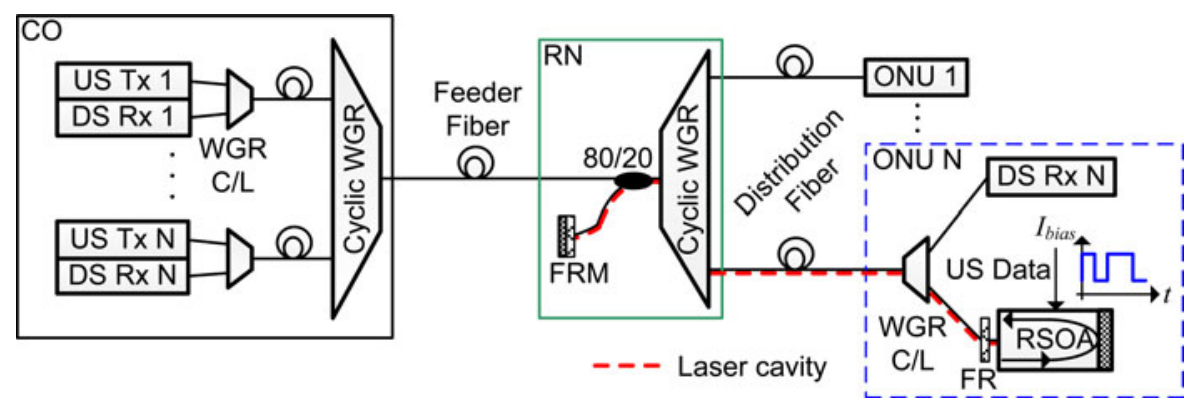

Fig. 1. RSOA-FCL based self-seeded WDM-PON Architecture.

The wave equation for the counter propagating signal fields is written as

$$
\begin{aligned}
& \frac{\partial E_{k}^{ \pm}(z, t)}{\partial z} \mp \frac{1}{v_{g}} \frac{\partial E_{k}^{ \pm}(z, t)}{\partial t} \\
& \quad=\frac{1}{2} E_{k}^{ \pm}(z, t)\left[-\gamma_{\mathrm{int}}+\Gamma \frac{g_{k}(N)}{1+\varepsilon_{\mathrm{NL}} P_{\mathrm{tot}}(z, t)}\left(1-j \alpha_{H}\right)\right]
\end{aligned}
$$

where $P_{\text {tot }}(z, t)$ is the total power at the position $z$ and time $t$ given by

$$
\begin{aligned}
P_{\text {tot }}(z, t)=\sum_{k}\left[\mid E_{k}^{+}(z, t)\right. & \left.+\left.E_{k}^{-}(z, t)\right|^{2}\right] \\
& +\sum_{j}\left[\left|\varepsilon_{j}^{+}(z, t)+\varepsilon_{j}^{-}(z, t)\right|^{2}\right] .
\end{aligned}
$$

$\gamma_{\mathrm{int}}$ and $\alpha_{H}$ are the internal scattering loss of the active region and the linewidth enhancement factor or the Henry factor, respectively. Since the lasing field extends over a spectral width of at most $100 \mathrm{GHz}$ within the OBPF, this paper assumes a constant $\alpha_{H}$-factor. Such an assumption is reasonable and allows for a sufficiently accurate SOA simulation [15], [16].

The equation for the ASE field is given by

$$
\begin{aligned}
\frac{\partial \varepsilon_{j}^{ \pm}(z, t)}{\partial z}=\frac{1}{2}\left[-\gamma_{\mathrm{int}}+\Gamma g_{j}(N(z, t))\right] & \varepsilon_{j}^{ \pm}(z, t) \\
& +R_{\mathrm{sp}, j}(N(z, t)) .
\end{aligned}
$$

In (8), the first part of the right hand side defines the amplification of the ASE as it propagates, whereas the second part is the spontaneous emission rate $R_{\mathrm{sp}}$ given as

$$
\begin{aligned}
& R_{\mathrm{sp}, j}(N) \\
& =\sqrt{\Gamma n_{\mathrm{sp}} g_{j}(N(z, t)) M \Delta v_{\mathrm{FSR}} h v_{j} \Delta z^{-1}}\left(\frac{x_{1}+j x_{2}}{\sqrt{2}}\right) .
\end{aligned}
$$

The spontaneous emission is assumed to have white Gaussian noise behavior [17]. $x_{1}$ and $x_{2}$ are Gaussian distributed random number sequences with mean zero and a variance of one. $n_{\mathrm{sp}}$ and $v_{j}$ are the inversion factor and the frequency of the $j$ th ASE mode, respectively. The spontaneous emission is calculated within the frequency range $M \Delta v_{\mathrm{FSR}}$ where $\Delta v_{\mathrm{FSR}}$ is the free spectral range (FSR) of the RSOA-FCL and $M$ is an integer number. In the next subsection we will discuss both.

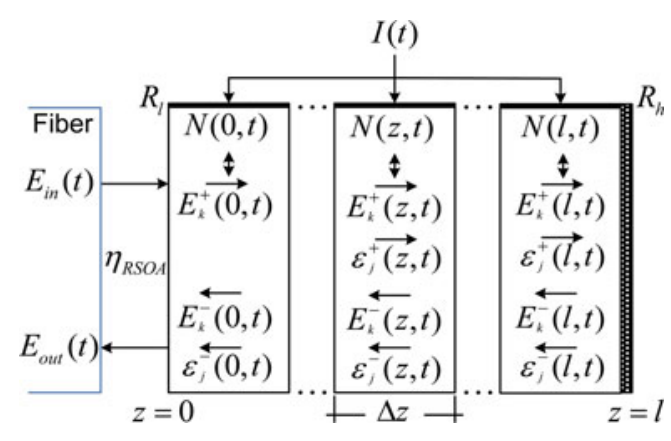

Fig. 2. RSOA simulation schematics where the counter propagating signal and ASE fields and carrier density are spatially resolved. The symbols are defined in the text.

To represent the propagation of the signal and ASE, the time $t$ and position $z$ change with steps

$$
\Delta z=\frac{l}{m}, \quad \Delta t=\frac{\Delta z}{v_{g}}
$$

where $l$ is the length of the RSOA active region with effective refractive index $n_{g} \cdot v_{g}=c / n_{g}$ is the group velocity, where $c$ is the speed of light in vacuum. The carrier density is numerically solved using the modified Euler method as in [16], [18].

The following boundary conditions are applied at the output and reflective facets of the RSOA shown in Fig. 2:

$$
\begin{aligned}
E_{k}^{+}(0, t) & =\sqrt{R_{l}} E_{k}^{-}(0, t)+\sqrt{\eta_{\mathrm{RSOA}}\left(1-R_{l}\right)} E_{\mathrm{in}, k}(t) \\
E_{k}^{-}(L, t) & =\sqrt{R_{h}} E_{k}^{+}(l, t) \\
\varepsilon_{j}^{+}(0, t) & =\sqrt{R_{l}} \varepsilon_{j}^{-}(0, t) \\
\varepsilon_{j}^{-}(L, t) & =\sqrt{R_{h}} \varepsilon_{j}^{+}(l, t) .
\end{aligned}
$$

$E_{\text {in }, k}(t)$ is the input field for the $k$ th signal mode whereas $R_{l}$ and $R_{h}$ are the power reflectivity of the output and the mirror facet, respectively. The coupling from fiber to the RSOA is given by $\eta_{\mathrm{RSOA}}$. The output signal and ASE are

$$
\begin{aligned}
E_{\text {out }, k}(t) & =\sqrt{\eta_{\mathrm{RSOA}}\left(1-R_{l}\right)} E_{k}^{-}(0, t) \\
\varepsilon_{\text {out }, j}(t) & =\sqrt{\eta_{\mathrm{RSOA}}\left(1-R_{l}\right)} \varepsilon_{j}^{-}(0, t) .
\end{aligned}
$$

\section{B. Cavity Model}

The simulation setup of the RSOA-FCL is shown in Fig. 3. The overall cavity length includes the distribution fiber and 


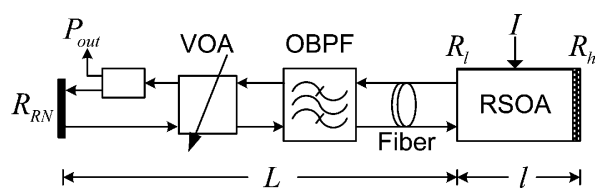

Fig. 3. Reflective SOA fiber-cavity laser (RSOA-FCL) formed by the RSOA mirror and the RN mirror. The cavity consists of the distribution fiber, an OBPF and VOA.

the RSOA. An OBPF mimics the WGR at the RN and performs frequency selection whereas a variable optical attenuator (VOA) represents the total insertion losses of all the passive constituents in the cavity.

The frequency response of the fiber assuming only group velocity dispersion and attenuation is

$$
H_{\mathrm{fbr}}(v)=\exp \left[\left(-0.1152 \alpha_{\mathrm{dB} / \mathrm{km}}+j \pi D \frac{\lambda_{o}^{2}}{c}\left(v-v_{o}\right)^{2}\right) L\right]
$$

where $\alpha_{\mathrm{dB} / \mathrm{km}}, D$ and $\lambda_{0}$ are the fiber attenuation, CD parameter and the center wavelength, respectively. The OBPF has a super Gaussian shape. Combining the Gaussian filter's frequency response and VOA attenuation gives

$$
\left|H_{\mathrm{OBPF}}(v)\right|=\alpha_{\mathrm{VOA}} \exp \left(-\frac{1}{2}\left(\frac{v-v_{0}}{\Delta u}\right)^{2 n}\right)
$$

where

$$
\Delta v=\frac{v_{3 \mathrm{~dB}}}{2 \sqrt[2 n]{\ln 2}}
$$

with $v_{3 \mathrm{~dB}}$ and $n$ being the $3 \mathrm{~dB}$ bandwidth and the order of the Gaussian filter, respectively, whereas $\alpha_{\mathrm{VOA}}$ is the single direction attenuation of the VOA.

The ASE and signal of the laser, which are now transmitted into the network are

$$
\begin{aligned}
\varepsilon_{\text {out }}(t) & =\sqrt{\eta_{\mathrm{cpr}}} F^{-1}\left\{H_{\mathrm{fbr}}(v) H_{\mathrm{OBPF}}(v) \cdot \tilde{\varepsilon}_{\text {out }}\right\} \\
E_{\text {out }, \mathrm{tx}}(t) & =\sqrt{\eta_{\mathrm{cpr}}} F^{-1}\left\{H_{\mathrm{fbr}}(v) H_{\mathrm{OBPF}}(v) \cdot \tilde{E}_{\text {out }}\right\}
\end{aligned}
$$

where $\eta_{\text {cpr }}$ is the ratio of the out coupled power. $\tilde{E}_{\text {out }}$ and $\tilde{\varepsilon}_{\text {out }}$ are the Fourier transforms of the output signal and ASE from the RSOA, respectively. $F^{-1}\{\}$ stands for inverse Fourier transform.

Now that the resonator cavity is explained in detail, we have to discuss the formation of the multiple longitudinal modes. The FSR of the mode, $\Delta v_{\mathrm{FSR}}$ is given by

$$
\Delta v_{\mathrm{FSR}}=\frac{c}{2\left(n_{\mathrm{fbr}} L+n_{g} l\right)}
$$

where $n_{\mathrm{fbr}}$ is the refractive index of the optical fiber. Since the RSOA-FCL extends up to tens of kilometer, the FSR is in the order of kHz. In other words, for an exemplary OBPF bandwidth of $100 \mathrm{GHz}$ we expect excited modes in the order of $10^{6}$. Thus solving the wave equation for all modes would be impractical. Similar to [14] we bundle neighboring modes to relieve the simulation load. We introduce $M$ as the integer number of modes bundled together and for which a single equation is solved. The (a)

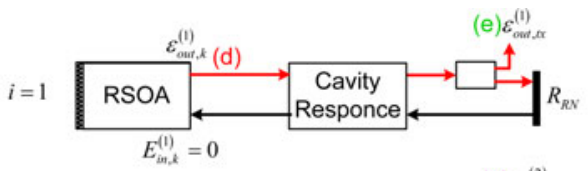

(b)
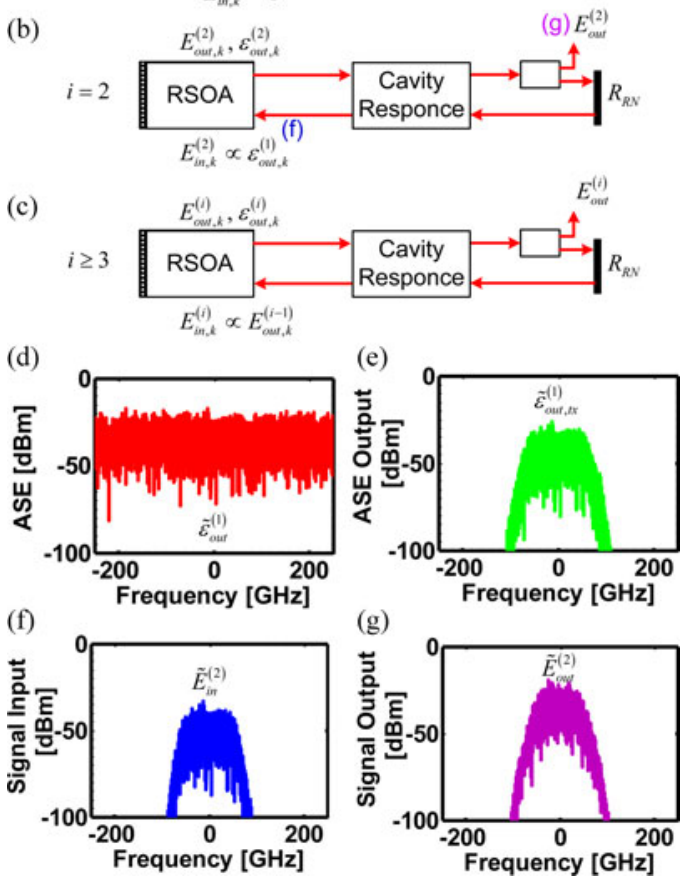

Fig. 4. Simulation procedure followed to simulate the modes in the RSOAFCL: (a) First iteration $i=1$ : the RSOA emits ASE shown in (d) while the input signal is zero (black arrow). The ASE coupled out of the RSOA-FCL and having traveled once through the fiber cavity and the WGR filter is shown in (e); (b) Second iteration $i=2$ : the scaled version of the ASE is fed into the RSOA as a signal, shown in $(\mathrm{g})$. The output signal of the RSOA-FCL laser is shown in (f); (c) After the third step $i \geq 1$ the signal is again fed into the cavity and a new iteration is started.

signal and ASE mode bundle frequencies, are then given by

$$
v_{k}=v_{0} \pm k M \Delta v_{\mathrm{FSR}}, \quad k=1,2, \ldots\left(N_{m}-1\right) / 2
$$

where $N_{m}$ and $v_{0}$ are the total number of modes and the center frequency, respectively.

\section{Simulation Results}

This section presents the simulation procedure and the results obtained for the RSOA-FCL. Initially we describe the ASE selfseeding within the cavity. Then, we report on the buildup of the laser emission and its spectral and temporal properties when the RSOA is unmodulated and modulated, respectively.

\section{A. Simulation Procedure}

The iteration steps of the signal and ASE fields in the RSOAFCL are summarized in Fig. 4. In a first iteration step, $i=1$ shown in Fig. 4(a), the RSOA just emits ASE as shown in Fig. 4(d). Subsequently this ASE light is traveling through the cavity undergoing dispersion and being filtered by the WGR. A monitor shows the spectrum in the RN, which can be seen in Fig. 4(e). In the second iteration, $i=2$ shown in Fig. 4(b), the ASE in the fiber cavity is reflected at the RN mirror and an attenuated fraction is fed back into the fiber, the WGR and 
into the RSOA as an input signal field, shown in Fig. 4(g). This signal is now amplified by the RSOA and passes through the fiber and OBPF to be coupled out, as depicted in Fig. 4(f). Here, the ASE spectral components with index $j$ are coupled to the signal with index $k$, i.e. $j=k$. The initial conditions for the first two iterations are given as

$$
\begin{aligned}
& \tilde{E}_{\mathrm{in}, k}^{(1)}=0 \\
& \tilde{E}_{\mathrm{in}, k}^{(2)}=\sqrt{R_{\mathrm{RN}}\left(1-\eta_{\mathrm{cpr}}\right)} \cdot \tilde{\varepsilon}_{\mathrm{out}, k}^{(1)} \cdot\left(H_{\mathrm{fbr}}\left(v_{k}\right) H_{\mathrm{OBPF}}\left(v_{k}\right)\right)^{2}
\end{aligned}
$$

where $R_{\mathrm{RN}}$ is the $\mathrm{RN}$ mirror reflectivity.

The RSOA output passes through the fiber and is filtered twice, thus the fiber and OBPF responses are squared as in (19). After the second iteration, the signal power significantly depletes the carriers and the ASE power noticeably diminishes. Therefore, for $i \geq 3$, we can neglect the coupling between the signal and ASE. The input signal field is then the RSOA output signal result from the previous iteration after passing through the channel twice, as per Fig. 4(c). It is given by

$$
\begin{aligned}
& \tilde{E}_{\mathrm{in}, k}^{(i)}=\sqrt{R_{\mathrm{RN}}\left(1-\eta_{\mathrm{cpr}}\right)} \\
& \cdot \tilde{E}_{\mathrm{out}, k}^{(i-1)} \cdot\left(H_{\mathrm{fbr}}\left(v_{k}\right) H_{\mathrm{OBPF}}\left(v_{k}\right)\right)^{2} .
\end{aligned}
$$

\section{B. Simulation of Unmodulated Operation}

Here, we investigate the RSOA-FCL operation when a DC bias current is applied to the RSOA and the modulation is disabled. This allows us to observe the laser spectrum and intensity waveform and to initially judge if such a highly multi-mode source could be used to transmit information at all. The RSOA and cavity parameters used for the system simulations are listed in Table I and they are based on [9] and further fitting to experimental results.

At first, we consider an RSOA-FCL with a short single mode fiber (SMF) of $1 \mathrm{~m}$ length and a dispersion parameter $D=$ $16 \mathrm{ps} /(\mathrm{nm} \cdot \mathrm{km})$. The evolution and the narrowing of the spectral width as the signal iterates in the cavity is depicted in Fig. 5. The output spectra after the tenth and 100th iteration are shown in the insets. The $10 \mathrm{~dB}$ spectral width is recorded after every fifth iteration up to the 100th iteration. The RSOA is biased with a $120 \mathrm{~mA}$ dc current and the overall bidirectional cavity loss is $16 \mathrm{~dB}$. This includes the insertion losses when twice passing through the WGR, the RN mirror and the fiber-to-RSOA coupling losses.

It can be seen that initially the spectral width of the laser is determined by the filter bandwidth, as plotted in Fig. 4(e). However, after a few iterations, the laser field builds up and mode competition leads to a narrowing of the spectrum. Fig. 5 shows how the spectral width of the long cavity laser narrows relatively quickly within the first 40 iterations. The Gaussian OBPF with a $3 \mathrm{~dB}$ bandwidth of $100 \mathrm{GHz}$ attenuates the outermost modes, which die out after only a few iterations. The RSOA-FCL gradually settles with less than $20 \mathrm{GHz}$ spectral width and a red shifted spectral peak relative to the center of

\begin{tabular}{|c|c|c|}
\hline Symbol & Quantity & Value \\
\hline$a_{0}$ & Differential gain & $2 \times 10^{-20} \mathrm{~m}^{-1}$ \\
\hline$\gamma_{\text {int }}$ & Internal scattering loss & $5000 \mathrm{~m}^{-1}$ \\
\hline$\alpha$ & Linewidth enhancement factor & 4 \\
\hline$\varepsilon_{N L}$ & Nonlinear gain coefficient & $3 \mathrm{~W}^{-1}$ \\
\hline$A$ & Nonradiative recombination & $4 \times 10^{8} \mathrm{~s}^{-1}$ \\
\hline $\mathrm{B}$ & Radiative recombination & $1 \times 10^{-16} \mathrm{~m}^{3} \mathrm{~s}^{-1}$ \\
\hline $\mathrm{C}$ & Auger Recombination & $1 \times 10^{-42} \mathrm{~m}^{6} \mathrm{~s}^{-1}$ \\
\hline$n_{s p}$ & Inversion factor & 2 \\
\hline$n_{g}$ & $\begin{array}{l}\text { Effective group refractive } \\
\text { index of the RSOA }\end{array}$ & 3.45 \\
\hline$N_{0}$ & Transparency carrier density & $5 \times 10^{23} \mathrm{~m}^{-3}$ \\
\hline$l$ & RSOA active region length & $1 \times 10^{-3} \mathrm{~m}$ \\
\hline$w$ & RSOA active region width & $2 \times 10^{-6} \mathrm{~m}$ \\
\hline$d$ & RSOA active region height & $0.1 \times 10^{-6} \mathrm{~m}$ \\
\hline$\Gamma$ & Confinement factor & 0.2 \\
\hline$R_{l}$ & Output facet reflectivity & $1 \times 10^{-6}$ \\
\hline$R_{h}$ & Mirror facet reflectivity & 0.2 \\
\hline$v_{3 d B}$ & OBPF bandwidth & $100 \mathrm{GHz}$ \\
\hline$n$ & OBPF order & 2 \\
\hline$\alpha_{d B / k m}$ & Fiber attenuation & $0.2 \mathrm{~dB} / \mathrm{km}$ \\
\hline$\lambda_{\theta}$ & Center frequency & $1550 \mathrm{~nm}$ \\
\hline$R_{R N}$ & RN mirror reflectivity & 0.86 \\
\hline$m$ & Spatial sections & 20 \\
\hline$N_{m}$ & Total number of modes & 966 \\
\hline$\eta_{R S O A}$ & RSOA coupling loss & $3 \mathrm{~dB}$ \\
\hline$\eta_{c p r}$ & Cavity network coupling ratio & 0.2 \\
\hline
\end{tabular}
the OBPF. The redshift can be seen in the insets of Fig. 5. One
TABLE I

RSOA-FIBER LASER COMPONENT PARAMETERS [9]

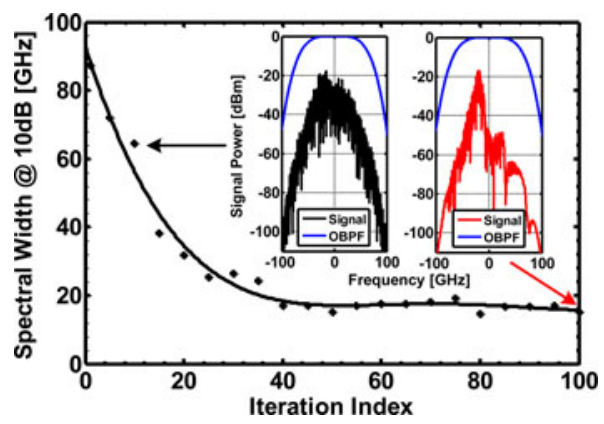

Fig. 5. Evolution of the signal spectral width versus iteration index in the $1 \mathrm{~m}$ long SMF cavity of the RSOA-FCL; the insets show sample spectra for the tenth and 100th iteration with OBPF frequency response (blue line).

might think that the laser spectra should be centered at the OBPF transmission maximum; however, this is not the case. The lasing spectrum extends to the redshifted transition band of the OBPF due to the Bogatov effect [19]. The dominance of the redshifted components implies that they receive more gain and thus lasing at these modes dominates. A further redshift of the peak at the low frequency edge of the WGR passband is prevented by an increased attenuation [20].

In addition, a stable output power waveform is observed because RSOA gain saturation suppresses fast intensity fluctuations of the seeding ASE and beating between the laser modes. This will be discussed in more detail below.

The simulation parameters for the RSOA listed in Table I were fitted to the actual data by comparing the simulation and experimental results of the power versus bias current $(P-I)$ plot. The $P-I$ plot of the RSOA-FCL with 6,11 , and $16 \mathrm{~dB}$ overall 


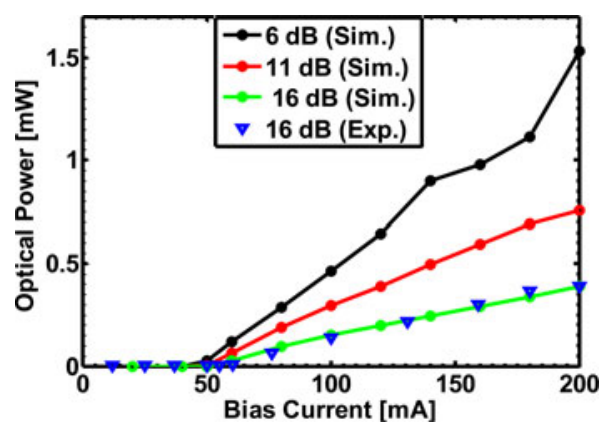

Fig. 6. Simulation and experimental plot of the output power versus bias current for the RSOA-FCL with $1 \mathrm{~m} \mathrm{SMF}$ cavity and 6, 10 and $16 \mathrm{~dB}$ overall cavity losses. The output power reduces for larger cavity loss.

cavity loss is depicted in Fig. 6 . The $P-I$ plot with a $16 \mathrm{~dB}$ loss is shown for both simulation and experiment. The experimental setup is modelled as the scheme depicted in Fig. 3, the only difference being that the OBPF is a Finisar WaveShaper that was programmed to mimic a $110 \mathrm{GHz}$ Gaussian bandpass filter. As expected, the output power diminishes for larger cavity losses. The threshold current is observed around $50 \mathrm{~mA}$ both in the simulation and in the experiment.

Subsequently, the long fiber cavity that may extend up to tens of kilometers results in a large number of longitudinal modes within the $100 \mathrm{GHz}$ bandwidth of the OBPF. Due to the broad spectrum, there is a strong effect of $\mathrm{CD}$ on the system performance. We then investigated two types of cavities with SMF and dispersion shifted fiber (DSF), where the SMF was simulated with $D=16 \mathrm{ps} /(\mathrm{nm} \cdot \mathrm{km})$ and the DSF was simulated with $D=0 \mathrm{ps} /(\mathrm{nm} \cdot \mathrm{km})$. The signal spectral width as a function of the fiber cavity length for SMF and DSF are shown in Fig. 7(a). During the simulation, the program was executed 50 iterations beyond the steady state operation point and then the mean of the 50 spectral width values was taken. It can be seen how the signal spectrum abruptly broadens for longer dispersive cavities due to an accumulation of CD, while it remains constant for the DSF cavities. The finding agrees well with the measurement results shown by the black triangles for SMF and blue star for the DSF in the same plot.

Fig. 7(b)-(e) exemplify the CD induced power fluctuations and the spectral broadening. Fig. 7(b) and (d) show the temporal power and spectra for $10 \mathrm{~m} \mathrm{SMF}$ and DSF cavities, respectively. In both cases, once the laser field has built up, the intensity remains constant with only small fluctuations. The "small" thereby refers to the fact that it is small given the highly multimoded field. When the SMF cavity is short, the CD is insignificant and the nonlinear RSOA with strong gain saturation suppresses the power fluctuation of the seeding ASE. Thus, the power converges to an almost constant value with a narrow spectrum as for the DSF case. The spectra for the two cavities are similar to the steady-state spectrum of the $1 \mathrm{~m} \mathrm{SMF}$ cavity shown in the inset of Fig. 5. When the cavity is extended beyond $0.5 \mathrm{~km} \mathrm{SMF}$, the $\mathrm{CD}$ alters the delicate phase relation between the multiple modes that is required to achieve a constant intensity. Once these modes reach the RSOA, the modes interfere constructively and destructively creating strong power fluctuations that cannot be (a)

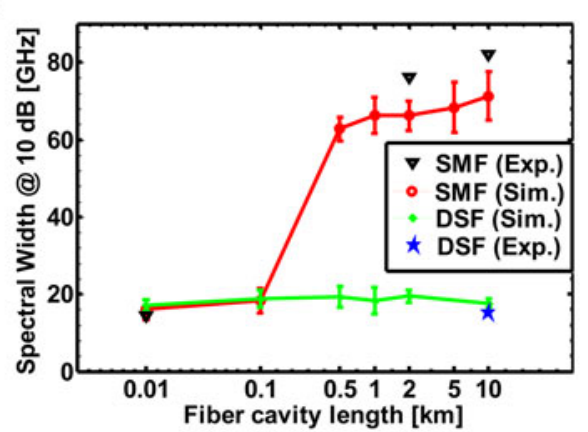

(b)
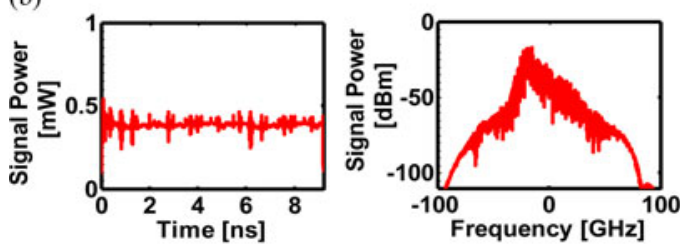

(c)
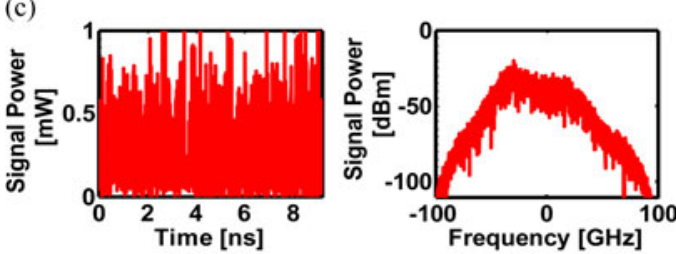

(d)
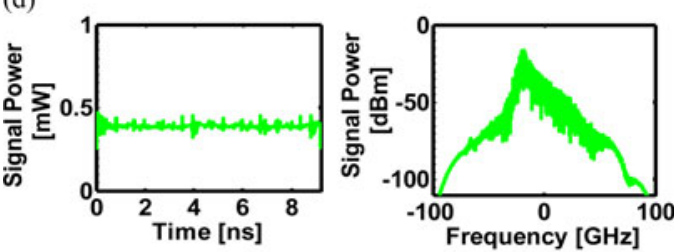

(e)
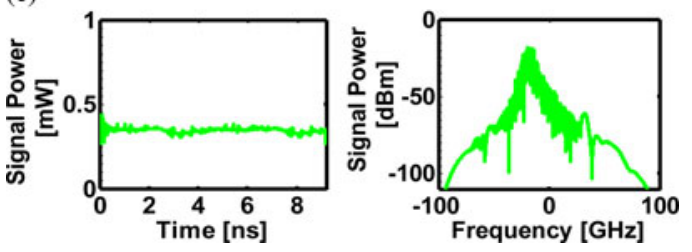

Fig. 7. Simulated and experimental spectral width as a function of the cavity length for SMF (red line) and DSF (blue line). The subfigures show simulated output powers with time (left) and spectra (right) for (b) $10 \mathrm{~m} \mathrm{SMF}$, (c) $1 \mathrm{~km}$ $\mathrm{SMF}$, (d) $10 \mathrm{~m} \mathrm{DSF}$ and (e) $1 \mathrm{~km}$ DSF cavities, respectively.

suppressed by the RSOA gain saturation; and thus leading to increased spectral broadening as depicted in Fig. 7(c). This CD induced mode partition noise substantially increases the relative intensity noise (RIN) of the laser and renders the laser useless for direct modulation of an US signal. However, when using a $1 \mathrm{~km}$ DSF cavity the phase relation of the modes is mostly preserved and a relatively flat output power with a narrow spectrum is obtained, as shown in Fig. 7(e).

It is instructive to plot the total output power of the RSOAFCL against the power in different spectral ranges that contribute to this total power. In Fig. 8(a) and (b) we show the power of 90 longitudinal mode bundles and the total power for a $10 \mathrm{~m} \mathrm{SMF}$ cavity RSOA-FCL. The power in a mode bundle is obtained by filtering the output field with a Lorentzian filter $(517 \mathrm{MHz}$ 


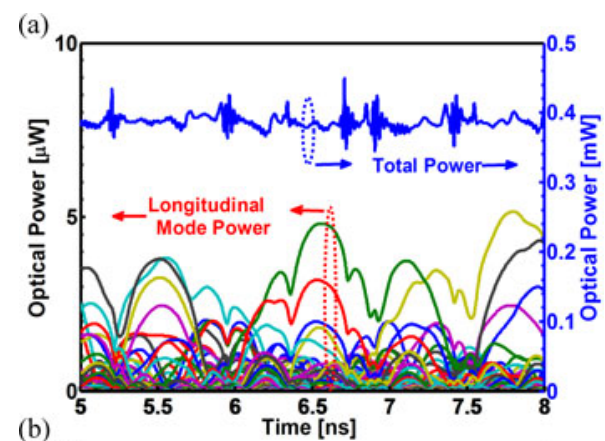

(b)

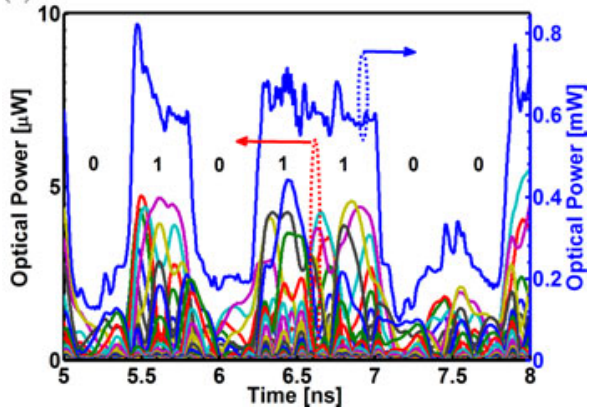

Fig. 8. Plot for the power of individual longitudinal mode bundles (colored lines) and the total power (blue line) for $10 \mathrm{~m} \mathrm{SMF} \mathrm{cavity} \mathrm{RSOA-FCL;}$ (a) without direct modulation and (b) with direct modulation at $2.5 \mathrm{Gbit} / \mathrm{s}$.

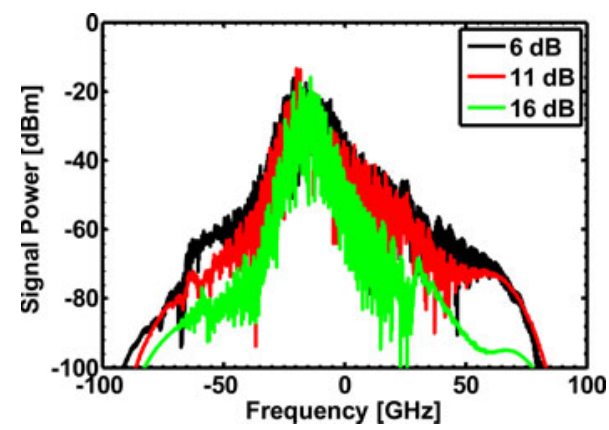

Fig. 9. Output signal spectra of RSOA-FCL with $1 \mathrm{~m}$ SMF cavity and 6, 11 and $16 \mathrm{~dB}$ overall cavity losses. The low cavity loss has broader spectrum due to the stronger multimode interaction.

full width at half maximum) centered at the modal frequencies. In Fig. 8(a), the colored lines show how the individual mode powers fluctuate due to strong mode competition while the total power (blue line) is relatively constant. Fig. 8(b) then shows that the fluctuations in the total output power remain low even if the laser is directly modulated. The modes still compete however; the output power now depends on the extinction ratio of the modulated bias current.

The cavity loss is another important parameter in the characterization of the RSOA-FCL. To observe the impact of cavity losses alone, we simulate a cavity with $1 \mathrm{~m}$ long SMF having negligible $\mathrm{CD}$ and the output spectra for 6,11 and $16 \mathrm{~dB}$ overall cavity losses are depicted in Fig. 9. For low cavity losses or strong feedback into the RSOA, the spectrum is broadened due to the interaction between the strong multiple modes reflected back into the RSOA.
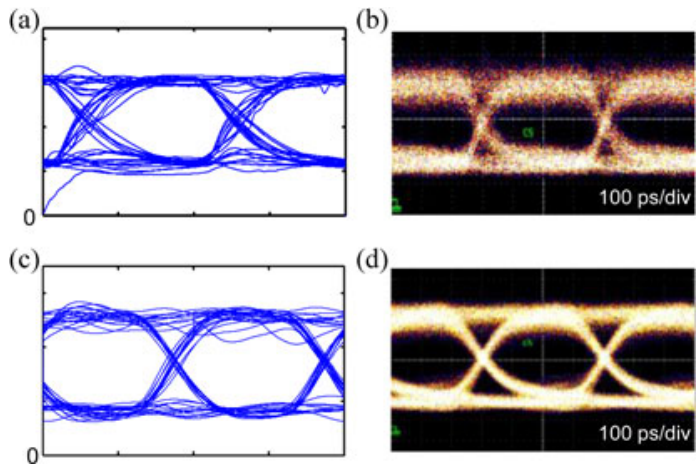

Fig. 10. Simulated [(a) and (c)] and measured [(b) and (d)] eye diagrams for $2.5 \mathrm{Gbit} / \mathrm{s}$ direct modulation of the RSOA-FCL with; (a) and (b) $10 \mathrm{~m} \mathrm{SMF}$ cavity; (c) and (d) $420 \mathrm{~m}$ SMF cavity.

\section{Simulation of Directly Modulated Laser}

This subsection presents the performance of the RSOA-FCL when used as a directly modulated transmitter. After the CW output signal reaches steady state a modulated bias current is supplied to the RSOA to encode data for US communications. A non-return to zero signal is encoded on the bias current, and for every iteration, a new pseudo random bit sequence (PRBS) is generated. This allows us to show explicitly that the RSOA with saturated gain simultaneously suppresses the recirculating residual intensity modulation from US communications in a previous iteration while encoding new information onto the field for new US transmission.

The eye diagrams for a $2.5 \mathrm{Gbit} / \mathrm{s}$ modulated RSOA-FCL are depicted in Fig. 10. The RSOA-FCL is biased at $120 \mathrm{~mA}$, and the modulation component swings between 70 and $170 \mathrm{~mA}$. The minimum current is $20 \mathrm{~mA}$ above the laser threshold. This corresponds to $6 \mathrm{~dB}$ extinction ratio in the output laser power. The ideal operation point that allows erasure of previous US signal is at a reduced extinction ratio as outlined in more detail in [9]. The bidirectional cavity loss is $11 \mathrm{~dB}$. Fig. 10(a) and (b) show simulation and experimental eye diagrams for a $10 \mathrm{~m}$ SMF cavity whereas (c) and (d) show simulation and experimental eye diagrams for a $420 \mathrm{~m}$ SMF cavity, respectively. The eyes are open since the cavities are relatively short and both $\mathrm{CD}$ and loss are low. Moreover, now the RSOA input for the $i$ th iteration is the modulated output signal from the $(i-1)$ th iteration having different PRBS. To encode a new data on the signal field, the modulation from the previous iteration must be suppressed. Thus, the open eyes in Fig. 10 are clear indications that the simultaneous modulation cancellation and remodulation operations are working perfectly.

In Fig. 11(a), the simulated cavity is extended to $10 \mathrm{~km}$ of SMF and it can be seen that the eye is completely closed. The accumulated $\mathrm{CD}$ impairment is responsible for the poor performance. On the other hand, if $10 \mathrm{~km}$ DSF replaces the SMF fiber, open eyes are obtained as shown in Fig. 11(b), though with less power due to the additional fiber attenuation.

Fig. 12 shows the change of the signal spectral width with the bit rates for $10 \mathrm{~m} \mathrm{SMF}$ and $1 \mathrm{~km}$ DSF. The plot illustrates that there is some small spectral broadening with higher bit-rates. 
(a)

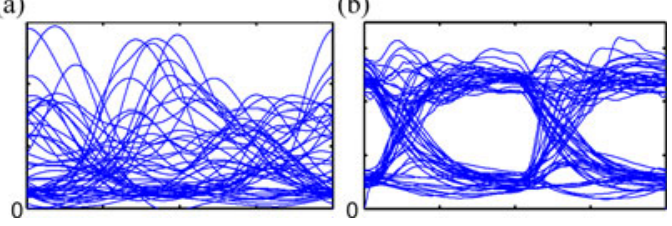

Fig. 11. Simulated eye diagrams for $2.5 \mathrm{Gbit} / \mathrm{s}$ direct modulation of the RSOAFCL with; (a) $10 \mathrm{~km} \mathrm{SMF} \mathrm{cavity} \mathrm{and} \mathrm{(b)} 10 \mathrm{~km}$ DSF cavity. The CD in the $10 \mathrm{~km}$ SMF results in complete closure of the eyes.

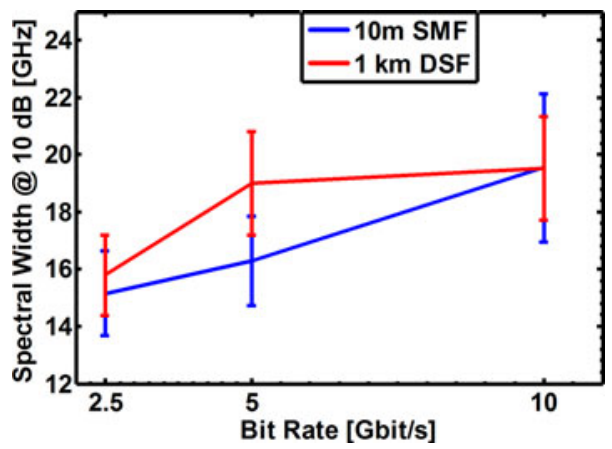

Fig. 12. Signal spectral width of the RSOA-FCL with $10 \mathrm{~m} \mathrm{SMF}$ and $1 \mathrm{~km}$ DSF cavities modulated at $2.5,5$ and $10 \mathrm{Gbit} / \mathrm{s}$.

This may be due to the chirp as a result of direct modulation and suggests that the reach of RSOA-FCL transmitter might be slightly reduced when increasing the bit-rate [6].

\section{CONCLUSION}

This paper has presented a theoretical model for the RSOAFCL as a colorless transmitter for next generation WDM-PON access. The ASE self-seeded laser cavity was found to narrow the spectrum to a smaller subset of modes with an almost constant output power allowing for a good operation with short fiber cavities. However, for longer and in particular for cavities with large chromatic dispersion, the output power strongly fluctuates since $\mathrm{CD}$ breaks the delicate phase relation between the multiple modes that is required to achieve constant power. The RSOA then can no longer suppress these fast fluctuations and thus the obtained directly modulated signal presents very poor performance, as evidenced by an almost complete eye closure. However, when the dispersion in the cavity is sufficiently small, as with a DSF, clear open eye diagrams are obtained at $2.5 \mathrm{Gbit} / \mathrm{s}$. Thus proving that both direct modulation and cancellation of the modulation part of the recirculating signal could occur simultaneously even for cavity lengths of the order of $10 \mathrm{~km}$. Self-seeded RSOA-FCL, as evidenced by this paper, are thus promising colorless sources for beyond $2.5 \mathrm{Gbit} / \mathrm{s}$ access transmission when operated with proper CD management.

\section{ACKNOWLEDGMENT}

The authors thank P. Chanclou from Orange Labs and G. Gavioli and G. Parlador from Alcatel-Lucent Italia.

\section{REFERENCES}

[1] E. Wong, L. Ka Lun, and T. B. Anderson, "Directly modulated self-seeding reflective semiconductor optical amplifiers as colorless transmitters in wavelength division multiplexed passive optical networks," J. Lightw. Technol., vol. 25, no. 1, pp. 67-74, Jan. 2007.

[2] L. Marazzi, P. Parolari, R. Brenot, G. De Valicourt, and M. Martinelli, "Network-embedded self-tuning cavity for WDM-PON transmitter," Opt. Exp., vol. 20, pp. 3781-3786, 2012.

[3] M. Sang-Rok, K. Myeong Gyun, S. Byung-Il, and L. Chang-Hee, " $2.5 \mathrm{~Gb} / \mathrm{s}$ injection seeded DWDM - PON using feed forward noise suppression," in Proc. OptoElectronics Commun. Conf. Int. Conf. Photon. Switching, 2013, pp. 1-2.

[4] N. Nadarajah, L. Ka Lun, and A. Nirmalathas, "Upstream access and local area networking in passive optical networks using self-seeded reflective semiconductor optical amplifier," IEEE Photon. Technol. Lett., vol. 19, no. 19 , pp. $1559-1561$, Oct. 2007.

[5] J. M. Kang, T. Y. Kim, I. H. Choi, S. H. Lee, and S. K. Han, "Selfseeded reflective semiconductor optical amplifier based optical transmitter for up-stream WDM-PON link," IET Optoelectron., vol. 1, pp. 77-81, 2007.

[6] L. Marazzi, P. Parolari, M. Brunero, A. Gatto, M. Martinelli, R. Brenot, S. Barbet, P. Galli, and G. Gavioli, "Up to 10.7-Gb/s High-PDG RSOAbased colorless transmitter for WDM networks," IEEE Photon. Technol. Lett., vol. 25, no. 7, pp. 637-640, Apr. 2013.

[7] Q. Deniel, F. Saliou, L. Anet Neto, N. Genay, B. Charbonnier, D. Erasme, and P. Chanclou, "Up to $10 \mathrm{Gbit} / \mathrm{s}$ transmission in WDM-PON architecture using external cavity laser based on self-tuning ONU," presented at the Opt. Fiber Commun. Conf., Los Angeles, CA, USA, 2012.

[8] G. De Valicourt, D. Make, J. Landreau, M. Lamponi, G. H. Duan, P. Chanclou, and R. Brenot, "High gain $(30 \mathrm{~dB})$ and high saturation power $(11 \mathrm{dBm}) \mathrm{RSOA}$ devices as colorless ONU sources in long-reach hybrid WDM/TDM-PON architecture," IEEE Photon. Technol. Lett., vol. 22, no. 3, pp. 191-193, Feb. 2010

[9] S. Ó. Dúill, L. Marazzi, P. Parolari, R. Brenot, C. Koos, W. Freude, and J. Leuthold, "Efficient modulation cancellation using reflective SOAs," Opt. Exp., vol. 20, pp. B587-B594, 2012.

[10] R. Brenot, S. Barbet, D. Make, C. Fortin, F. Lelarge, F. Pommereau, F. Martin, and F. Poingt, "Evidence of gain modulation in km-long lasers," in Proc. 23rd IEEE Int. Semicond. Laser Conf., 2012, pp. 108-109.

[11] G. De Valicourt, D. Make, C. Fortin, A. Enard, F. Van-Dijk, and R. Brenot, "10 Gbit/s modulation of reflective SOA without any electronic processing," in Proc. Opt. Fiber Commun. Conf. Expo. Nat. Fiber Opt. Eng. Conf., 2011, pp. 1-3.

[12] M. Presi, A. Chiuchiarelli, R. Corsini, and E. Ciaramella, "Self-seeding of semiconductor lasers for next-generation WDM Passive Optical Networks," in Proc. 15th Int. Conf. Transparent Opt. Netw., 2013, pp. 1-4.

[13] M. Martinelli, L. Marazzi, P. Parolari, M. Brunero, and G. Gavioli, "Polarization in retracing circuits for WDM-PON," IEEE Photon. Technol. Lett., vol. 24, no. 14, pp. 1191-1193, Jul. 2012.

[14] M. J. Connelly, "Wideband semiconductor optical amplifier steady-state numerical model," IEEE J. Quantum Electron., vol. 37, no. 3, pp. 439-447, Mar. 2001.

[15] J. Leuthold, "Advanced indium-phosphide waveguide Mach-Zehnder interferometer all-optical switches and wavelength converters," Ph.D. Dissertation, Swiss Fed. Inst. Technol., Zurich, Switzerland, 1998.

[16] S. O'Duill, L. Marazzi, P. Parolari, W. Freude, C. Koos, and J. Leuthold, "Modulation cancellation properties of reflective SOAs," presented at the Eur. Conf. Exhib. Opt. Commun., Amsterdam, The Netherlands, 2012.

[17] A. M. De Melo and K. Petermann, "On the amplified spontaneous emission noise modeling of semiconductor optical amplifiers," Opt. Commun., vol. 281, pp. 4598-4605, 9/15/ 2008 .

[18] W. Jin, A. Maitra, C. G. Poulton, W. Freude, and J. Leuthold, "Temporal dynamics of the alpha factor in semiconductor optical amplifiers," $J$. Lightw. Technol., vol. 25, no. 3, pp. 891-900, Mar. 2007.

[19] A. P. Bogatov, P. G. Eliseev, and B. N. Sverdlov, "Anomalous interaction of spectral modes in a semiconductor laser," IEEE J. Quantum Electron., vol. 11, no. 7, pp. 510-515, Jul. 1975.

[20] S. S. Girard, M. M. Piche, C. Hongxin, G. G. Schinn, O. Wang-Yuhl, and B. B. Bouma, "SOA fiber ring lasers: single- versus multiple-mode oscillation," IEEE J. Select. Topics Quantum Electron., vol. 17, no. 6, pp. 1513-1520, Nov./Dec. 2011. 